\title{
On extensions of the Mittag-Leffler theorem
}

\author{
by EwA LigockA (Warszawa)
}

\begin{abstract}
The classical Mittag-Leffler theorem on meromorphic functions is extended to the case of functions and hyperfunctions belonging to the kernels of linear partial differential operators with constant coefficients.
\end{abstract}

1. Introduction and preliminaries. The aim of the present paper is to extend the classical Mittag-Leffler theorem on meromorphic functions to the case of functions belonging to the kernels of linear partial differential operators with constant coefficients.

We use the following convention:

(*) The expression "differential operator" stands for "linear partial differential operator with constant coefficients in $\mathbb{R}^{n}$ ".

We now formulate the following condition on sequences. Let $\varrho=\left(\varrho_{1}, \ldots\right.$ $\left.\ldots, \varrho_{n}\right) \in \mathbb{R}^{n}, \varrho_{i} \geq 1, i=1, \ldots, n$. Let $I=\mathbb{N}^{n}$ be the set of multiindices. A sequence $\left\{c_{\alpha}\right\}_{\alpha \in I}$ satisfies condition $\mathrm{A}_{\varrho}$ iff for every $\varepsilon>0$ there exists a constant $C_{\varepsilon}$ such that

$$
\left|c_{\alpha}\right| \leq C_{\varepsilon} \frac{\varepsilon^{|\alpha|}}{\alpha_{1}^{\varrho_{1} \alpha_{1}} \ldots \alpha_{n}^{\varrho_{n} \alpha_{n}}}, \quad \forall \alpha=\left(\alpha_{1}, \ldots, \alpha_{n}\right) \in I .
$$

If $\varrho=(1, \ldots, 1)$ then we say that the sequence $\left\{c_{\alpha}\right\}_{\alpha \in I}$ satisfies condition A. By Stirling's formula, condition A is equivalent to the following: for every $\varepsilon$ there exists a constant $C_{\varepsilon}^{\prime}$ such that

$$
\left|c_{\alpha}\right| \leq C_{\varepsilon}^{\prime} \frac{\varepsilon^{|\alpha|}}{\alpha_{1} ! \ldots \alpha_{n} !}, \quad \forall \alpha=\left(\alpha_{1}, \ldots, \alpha_{n}\right) \in I .
$$

Let us make one more convention.

1991 Mathematics Subject Classification: Primary 32A45, 35E20; Secondary 31B05, 31B30, 30B10.

Key words and phrases: hyperfunction, Laurent expansion, elliptic, polyharmonic, hypoelliptic, $P$-convex for supports. 
(**) $\Omega$ will always denote an open set in $\mathbb{R}^{n}$, and $\left\{x_{k}\right\}_{k \in \mathbb{N}}$ a discrete sequence of distinct points of $\Omega$.

We always denote by $B(\Omega)$ the space of hyperfunctions on $\Omega$ (see [9], $[7])$ and by $D^{\prime}(\Omega)$ the space of distributions on $\Omega$. Our work was inspired by the following theorem on hyperfunctions proved by Chung, Kim and Lee ([4], Theorem 2.6).

THEOREM 0. Let $P(D)$ be a differential operator $(*), U$ be the unit ball in $\mathbb{R}^{n}$ and $u$ be a hyperfunction on $U$. Assume that $P(D) u=0$ on $U \backslash\{0\}$. Then there exists a solution $v \in B(U)$ of the equation $P(D) v=0$ and a sequence $\left\{c_{\alpha}\right\}_{\alpha \in I}$ satisfying condition A such that

$$
u=v+\sum_{\alpha \in I} c_{\alpha} \partial^{\alpha} E,
$$

where $E$ is a fundamental solution of $P(D)$.

For elliptic differential operators similar theorems were proved earlier by Wachman [13] and Harvey and Polking [6]. These results were extended by Tarkhanov (see [10]-[12]) to the case of elliptic systems.

Nowadays, one usually thinks of the Mittag-Leffler theorem as of a theorem from Cech cohomology theory. This approach permits finding an analogue of it for elliptic complexes (see, for example, [12], 4.2.7).

However, in its original form the Mittag-Leffler theorem concerned the construction of a holomorphic function with a priori given singularities at a priori given isolated points. In the present note, we want to generalize this "constructive side" of the Mittag-Leffler theorem.

We prove a general Mittag-Leffler theorem for hyperfunctions and use it in the case of elliptic operators. We also consider the case of hypoelliptic operators using the results of [7].

\section{Mittag-Leffler theorem for hyperfunctions}

2.1. Theorem. Let $\Omega$ be an open set in $\mathbb{R}^{n}$ and let $P(D)$ be a differential operator $(*)$. Assume that $\left\{\Omega_{j}\right\}_{j \in S}$ is an open covering of $\Omega$ and hyperfunctions $u_{j} \in B\left(\Omega_{j}\right)$ are such that $\left.P(D)\left(u_{i}-u_{j}\right)\right|_{\Omega_{i} \cap \Omega_{j}}=0$. Then there exists a hyperfunction $u \in B(\Omega)$ such that for every $j \in S$,

$$
\left.P(D)\left(u-u_{j}\right)\right|_{\Omega_{j}}=0 .
$$

Proof. By the very definition of hyperfunction (see [9]) there exists a hyperfunction $v$ on $\Omega$ such that $\left.v\right|_{\Omega_{j}}=P(D) u_{j}$. By Corollary 1 in $\S 1$ of Chapter III of [9] there exists $u \in B(U)$ such that $P u=v$. Hence

$$
P\left(u-u_{j}\right)=v-P u_{j}=0 \quad \text { on } \Omega_{j} .
$$

2.2. Corollary. Let $P(D)$ be as above and let $\Omega$ and $\left\{x_{k}\right\}_{k \in \mathbb{N}}$ be as in $(* *)$. For each $k \in \mathbb{N}$ let $V_{k}$ be an open neighbourhood of $x_{k}$. Assume that 
$V_{i} \cap V_{j}=\emptyset$ if $i \neq j$. For every sequence $u_{j} \in B\left(V_{j}\right)$ with $\left.P u_{j}\right|_{V_{j} \backslash\left\{x_{j}\right\}}=0$ there exists $u \in B(\Omega)$ such that $\left.P(D) u\right|_{\Omega \backslash\left\{x_{1}, x_{2}, \ldots\right\}}=0$ and for each $j \in \mathbb{N}$, $\left.P(D)\left(u-u_{j}\right)\right|_{V_{j}}=0$.

Proof. Take $\Omega_{0}=\Omega \backslash\left\{x_{1}, x_{2}, \ldots\right\}, u_{0}=0, \Omega_{j}=V_{j}$ and use Theorem 2.1.

2.3. Remark. Note that by Theorem 0 each $u_{j}$ must be equal to $v_{j}+$ $\sum_{\alpha} c_{\alpha, j} \partial^{\alpha} E\left(x-x_{j}\right)$ where $\left\{c_{\alpha, j}\right\}_{\alpha \in I}$ satisfies condition $\mathrm{A}$ and $E$ is a fundamental solution for $P(D)$.

Corollary 2.2 yields immediately the following.

2.4. TheOREM (Mittag-Leffler theorem for hyperfunctions). Let $\Omega$ and $\left\{x_{k}\right\}_{k \in \mathbb{N}}$ be as in $(* *)$. For each $k \in \mathbb{N}$ let $V_{k}$ be an open neighbourhood of $x_{k}$ such that $V_{i} \cap V_{j}=\emptyset$ if $i \neq j$. Let $P(D)$ be a differential operator and let $E$ be its fundamental solution. For every $k \in \mathbb{N}$ take a sequence $\left\{c_{\alpha, k}\right\}_{\alpha \in I}$ satisfying condition A and define

$$
R_{k}(x)=\sum_{\alpha \in I} c_{\alpha, k} \partial^{\alpha} E(x) .
$$

There exists $u \in B(\Omega)$ such that $P(D) u=0$ on $\Omega \backslash\left\{x_{1}, x_{2}, \ldots\right\}$ and

$$
P(D)\left(u-R_{k}\left(x-x_{k}\right)\right)=0 \quad \text { on } V_{k} \text { for each } k \in \mathbb{N} \text {. }
$$

2.5. Remark. Let us return to Theorem 0. For a general differential operator $P(D)$ there is a problem of uniqueness of the Laurent expansion $\sum_{\alpha \in I} c_{\alpha} \partial^{\alpha} E$ for a given hyperfunction $u$. The situation is as follows. For a fixed $u \in B(U)$ this expansion is unique. If $u$ is defined on $U \backslash\{0\}$ and we extend it to a hyperfunction $\widetilde{u}$ on $U$ then the Laurent expansion of $\widetilde{u}$ on $U$ depends on the choice of $\widetilde{u}$. (This is why Theorem 2.5 of [4] is incorrectly stated and proved. Compare [6], [13].) However, the restriction of the Laurent expansion to $U \backslash\{0\}$ does not depend on the choice of $\widetilde{u}$. Let $\widetilde{u}_{1}$ and $\widetilde{u}_{2}$ be two such extensions. We have $\widetilde{u}_{1}-\widetilde{u}_{2}=\sum_{\alpha \in I} d_{\alpha} \partial^{\alpha} \delta_{0}$ and hence if $\widetilde{u}_{2}=v_{2}+\sum_{\alpha \in I} c_{\alpha} \partial^{\alpha} E$ then $u_{1}=v_{1}+\sum_{\alpha \in I} c_{\alpha} \partial^{\alpha} E+\sum_{\alpha \in I} d_{\alpha} P(D) \partial^{\alpha} E$. Hence the difference between the Laurent expansions for $\widetilde{u}_{1}$ and $\widetilde{u}_{2}$ has support contained in $\{0\}$.

The abstract Theorem 2.4 can be useful only if we have some knowledge about the fundamental solution of $P(D)$ and the regularity of solutions of the equation $P(D) u=0$.

3. The case of elliptic operators. Recall two characterizations of elliptic differential operators $(*)$. The ellipticity of $P(D)$ is equivalent to each of the following two conditions:

1) If $u \in B(\Omega)$ and $P(D) u=0$ then $u$ is a real-analytic function on $\Omega$. 
2) $P(D)$ has a fundamental solution $E$ which is real-analytic outside $\{0\}$ (see [7]).

Suppose now that $\left\{c_{\alpha}\right\}_{\alpha \in I}$ is a sequence satisfying condition A.

It follows from the Cauchy inequalities that if $h$ is a function real-analytic on $\Omega$ then so is $g=\sum_{\alpha \in I} c_{\alpha} \partial^{\alpha} h$.

Hence Theorem 2.4 yields the following

3.1. TheOREM. Let $P(D)$ be an elliptic differential operator and let $E$ be its fundamental solution in $\mathbb{R}^{n}$ real-analytic outside zero. Let $\Omega$ and $\left\{x_{k}\right\}_{k \in \mathbb{N}}$ be as in (**). For each $n \in \mathbb{N}$ take $\left\{c_{\alpha}\right\}_{\alpha \in I}$ satisfying condition $\mathrm{A}$ and define

$$
R_{k}(x)=\sum_{\alpha \in I} c_{\alpha} \partial^{\alpha} E(x)
$$

which is analytic on $\mathbb{R}^{n} \backslash\{0\}$. There exists an analytic function $u$ on $\Omega \backslash\left\{x_{1}, x_{2}, \ldots\right\}$ such that $P(D) u=0$ on $\Omega \backslash\left\{x_{1}, x_{2}, \ldots\right\}$, for each $k \in \mathbb{N}$, $u(x)-R_{k}\left(x-x_{k}\right)$ is analytic on some neighbourhood $V_{k}$ of $x_{k}$, and $P(D)\left(u(x)-R_{k}\left(x-x_{k}\right)\right)=0$ on $V_{k}$.

Theorem 3.1 is particularly interesting when we have an explicit formula for the fundamental solution $E$.

The most important cases are:

1) $P(D)=\frac{\partial}{\partial \bar{z}}$ on $\mathbb{C}\left(=\mathbb{R}^{2}\right), \quad E(z)=\frac{1}{\pi z}$,

2) $P(D)=\Delta=\sum_{i=1}^{n} \frac{\partial^{2}}{\partial x_{i}^{2}}, \quad E(x)= \begin{cases}-\ln |x|, & n=2, \\ c_{n} /|x|^{n-2}, & n>2,\end{cases}$

3) $P(D)=\Delta^{m}$,

$$
E(x)= \begin{cases}c_{m n}|x|^{2 m-n} \ln |x| & \text { if } 2 m \geq n \text { and } n \text { is even, } \\ c_{m, n} /|x|^{n-2 m} & \text { if } n \text { is odd or } n>2 m \text { and } n \text { is even }\end{cases}
$$

(see [1]).

3.2. ThEOREM (Mittag-Leffler theorem for holomorphic functions). Let $\Omega$ be an open set in $\mathbb{C}$ and let $\left\{z_{k}\right\}_{k \in \mathbb{N}}$ be a discrete sequence of distinct points of $\Omega$. For each $k \in \mathbb{N}$ put $R_{k}\left(z-z_{k}\right)=\sum_{i=1}^{\infty} a_{i} /\left(z-z_{k}\right)^{i}$. There exists a function $g(z)$ holomorphic on $\Omega \backslash\left\{z_{1}, z_{2}, \ldots\right\}$ such that the principal part of the Laurent expansion of $g$ at $z_{k}$ is equal to $R_{k}\left(z-z_{k}\right)$ for every $k \in \mathbb{N}$.

3.3. TheOREM (Mittag-Leffler theorem for harmonic functions). Let $\Omega$ and $\left\{x_{k}\right\}_{k \in \mathbb{N}}$ be as in $(* *)$. For each $k \in \mathbb{N}$ let $\sum_{j=0}^{\infty} P_{j, k}(x)$ be a series of $j$-homogeneous harmonic polynomials, convergent on $\mathbb{R}^{n}$, and let 


$$
R_{k}(x)= \begin{cases}\sum_{j=0}^{\infty} \frac{P_{j, k}(x)}{|x|^{2 j+n-2}} & \text { if } n>2, \\ \sum_{j=1}^{\infty} \frac{P_{j, k}(x)}{|x|^{2 j}}+c_{0} \ln |x| & \text { if } n=2 .\end{cases}
$$

There exists a function u harmonic on $\Omega \backslash\left\{x_{1}, x_{2}, \ldots\right\}$ such that for each $k \in \mathbb{N}, u(x)-R_{k}\left(x-x_{k}\right)$ is harmonic on some neighbourhood of $x_{k}$.

(Information on convergent Laurent series of harmonic functions can be found in [3], [2] and [5].)

3.4. Theorem (Mittag-Leffler theorem for polyharmonic functions). Let $\Omega$ and $\left\{x_{k}\right\}$ be as above. For each $k$ let $\sum_{j=0}^{\infty} P_{j, k}(x)$ be a series of $m$ polyharmonic polynomials, convergent on $\mathbb{R}^{n}$. Let

$$
R_{k}(x)=\sum_{j=0}^{\infty} \frac{P_{j, k}(x)}{|x|^{2 j+n-2 m}} \quad \text { if } n>2 m \text { or } n \text { is odd }
$$

and

$$
\begin{aligned}
R_{k}(x)=\sum_{j \geq 2 m-n}^{\infty} \frac{P_{j, k}(x)}{|x|^{2 j+n-2 m}} \sum_{|\alpha|=0}^{2 m-n} d_{\alpha, k} \partial^{\alpha}\left(|x|^{2 m-n} \ln |x|\right) \\
\text { if } 2 m \geq n \text { and } n \text { is even. }
\end{aligned}
$$

There exists a function $u, m$-polyharmonic on $\Omega \backslash\left\{x_{1}, x_{2}, \ldots\right\}$, such that for each $k \in \mathbb{N} u(x)-R_{k}\left(x-x_{k}\right)$ is m-polyharmonic on some neighbourhood of $x_{k}$.

3.5. REMARK. In the above formulas we have

$$
P_{j, k}=|x|^{2 j+n-2 m} \sum_{|\alpha|=j} c_{\alpha, k} \partial^{\alpha} E,
$$

where $\left\{c_{\alpha, k}\right\}$ satisfies condition A.

4. The case of hypoelliptic operators. The hypoelliptic differential operators $(*)$ can be characterized as those operators which have a fundamental solution $E, C^{\infty}$-smooth on $\mathbb{R}^{n} \backslash\{0\}$ (see [7], Vol. 2, Theorem 11.1.1).

There are two major obstacles to extending Theorem 3.1 to hypoelliptic operators. First, if $P(D)$ is hypoelliptic but not elliptic, then there exist a domain $\Omega \subset \mathbb{R}^{n}$ and $u \in B(\Omega)$ such that $P u=0$ on $\Omega$ and $u \notin D^{\prime}(\Omega)$ (see $[9])$.

Moreover, condition A on the sequence $\left\{c_{\alpha}\right\}$ is too weak to guarantee that $\sum_{\alpha} c_{\alpha} \partial^{\alpha} E$ is $C^{\infty}$-smooth on $\mathbb{R}^{n} \backslash\{0\}$. (If it is not $C^{\infty}$-smooth on $\mathbb{R}^{n} \backslash\{0\}$ then it does not belong to $D^{\prime}(\Omega)$.) 
Hence in order to get an analogue of Theorem 3.1 we must put additional assumptions on the sequence $\left\{c_{\alpha}\right\}$ and the open set $\Omega$. Those assumptions will depend on the operator $P(D)$.

4.1. Definition. Let $\varrho=\left(\varrho_{1}, \ldots, \varrho_{n}\right)$, with $\varrho_{i} \geq 1$ for all $i$. We say that $u \in C^{\infty}(\Omega)$ belongs to the class $\Gamma_{\varrho}(\Omega)$ iff for each compact $K \subset \Omega$ there exists $C_{K}$ such that for every $x \in K$ and multiindex $\alpha=\left(\alpha_{1}, \ldots, \alpha_{n}\right)$,

$$
\left|\partial^{\alpha} u(x)\right| \leq \frac{C_{K}^{|\alpha|+1}}{\alpha_{1}^{\varrho_{1} \alpha_{1}} \ldots \alpha_{n}^{\varrho_{n} \alpha_{n}}} .
$$

The class $\Gamma_{\varrho}(\Omega)$ is called the anisotropic Gevrey class.

The Stirling and Newton formulas imply the following.

4.2. Proposition. If $\left\{c_{\alpha}\right\}_{\alpha \in I}$ satisfies condition $\mathrm{A}_{\varrho}$ and $u \in \Gamma_{\varrho}(\Omega)$ then $\sum c_{\alpha} \partial^{\alpha} u \in \Gamma_{\varrho}(\Omega)$.

In Hörmander's book [7], Vol. 2, the following fact was proved:

$(* * *) \quad$ For every hypoelliptic operator $P(D)$ there exists $\varrho=\left(\varrho_{1}, \ldots, \varrho_{n}\right)$ (and an orthonormal system of coordinates in $\mathbb{R}^{n}$ ) such that if $u \in$ $D^{\prime}(\Omega)$ and $P(D) u=0$ then $u \in \Gamma_{\varrho}(\Omega)$ for every open set $\Omega$ in $\mathbb{R}^{n}$ (Theorem 11.4.12 of [7]).

Let us recall some more facts from Hörmander's book. Let $P(D)$ be a linear partial differential operator with constant coefficients and let $\Omega$ be an open set in $\mathbb{R}^{n}$. We say that $\Omega$ is P-convex for supports if for every compact $K \subset \Omega$ there exists a compact $K^{\prime} \subset \Omega$ such that $\varphi \in C_{0}^{\infty}(\Omega)$ and $\operatorname{supp} P(-D) \varphi \subset K$ implies that $\operatorname{supp} \varphi \subset K^{\prime}$.

A convex open domain is $P$-convex for supports for every $P$ and $K^{\prime}=$ conv $K$.

If $P(D)$ is as above and $\Omega$ is $P$-convex for supports then for every $v \in$ $C^{\infty}(\Omega)$ there exists $u \in C^{\infty}(\Omega)$ such that $P(D) u=v$ ([7], Theorem 10.6.7 and Corollary 10.6.8).

We are now in a position to prove

4.3. Theorem. Let $P(D)$ be a differential hypoelliptic operator. Let $\varrho=$ $\left(\varrho_{1}, \ldots, \varrho_{n}\right)$ be as in $(* * *)$. Assume that $\Omega$ is $P$-convex for supports and that $\left\{x_{k}\right\}$ is a discrete sequence of distinct points in $\Omega$. For each $k \in \mathbb{N}$ let $R_{k}=\sum_{\alpha} c_{\alpha, k} \partial^{\alpha} E$, where $E$ is a fundamental solution for $P(D), C^{\infty}$ smooth on $\mathbb{R}^{n} \backslash\{0\}$, and $\left\{c_{\alpha, k}\right\}$ satisfies condition $\mathrm{A}_{\varrho}$ for each $k$. Then there exists $u \in \Gamma_{\varrho}\left(\Omega \backslash\left\{x_{1}, x_{2}, \ldots\right\}\right)$ such that $P(D) u=0$ and for each $k \in \mathbb{N}$ there exist $\Omega \supset V_{k} \ni x_{k}$ such that $u(x)-R_{k}\left(x-x_{k}\right) \in \Gamma_{\varrho}\left(V_{k}\right)$ and $P(D)\left[u(x)-R_{k}\left(x-x_{k}\right)\right]=0$ on $V_{k}$. 
Proof. For every $k$ take $V_{k}^{\prime}=B\left(x_{k}, r_{k}\right) \subset \Omega$ such that $V_{i}^{\prime} \cap V_{j}^{\prime}=\emptyset$ for $i \neq j$. Let $V_{k}=B\left(x_{k}, r_{k} / 2\right)$. Let $\varphi_{k}$ be a $C^{\infty}$ function such that $\varphi_{k}=0$ on $\Omega \backslash V_{k}^{\prime}$ and $\varphi_{k}=1$ on $V_{k}$.

Proposition 4.2 implies that $R_{k}\left(x-x_{k}\right) \in \Gamma_{\varrho}\left(\mathbb{R}^{n} \backslash\left\{x_{k}\right\}\right)$. Note that $P(D)\left[R_{k}\left(x-x_{k}\right)\right]=0$ in $\mathbb{R}^{n} \backslash\left\{x_{k}\right\}$. Thus $f(x)=\sum_{i=1}^{\infty} \varphi_{i} R_{k}\left(x-x_{i}\right) \in$ $C^{\infty}\left(\Omega \backslash\left\{x_{1}, x_{2}, \ldots\right\}\right)$. Let $g(x)=P(D) f(x)$ on $\Omega \backslash \bigcup_{i=1}^{\infty} V_{i}$ and $g(x)=0$ on $V_{i}$.

Since $g \in C^{\infty}(\Omega)$ there exists $v \in C^{\infty}$ such that $P(D) v=g$ on $\Omega$. Then $u=f-v \in C^{\infty}\left(\Omega \backslash\left\{x_{1}, x_{2}, \ldots\right\}\right)$ and $P(D) u=0$ on $\Omega \backslash\left\{x_{1}, x_{2}, \ldots\right\}$. On each $V_{k}, u-R_{k}\left(x-x_{k}\right)=-v \in C^{\infty}\left(V_{k}\right)$ and $P(D)\left(u-R_{k}\right)=0$. It follows from Theorem 11.4 .12 of [7] that $u \in \Gamma_{\varrho}\left(\Omega \backslash\left\{x_{1}, x_{2}, \ldots\right\}\right)$ and $u(x)-R_{k}\left(x-x_{k}\right) \in \Gamma_{\varrho}\left(V_{k}\right)$.

In order to characterize the isolated singularities of solutions of the equation $P(D) u=0$ we must know the answer to the following two open questions:

4.4. Problem. Assume that $u \in B(U), U$ is the unit ball in $\mathbb{R}^{n}, P(D) u$ $=0$ on $U$ and $\left.u\right|_{U \backslash\{0\}}$ is a $C^{\infty}$-smooth function on $U \backslash\{0\}$. Is it true that if $P(D)$ is hypoelliptic then $u$ is $C^{\infty}$-smooth on $U$ ?

4.5. Problem. Let $P(D)$ be a hypoelliptic differential operator. Assume that $P(D) f=0$ on $U \backslash\{0\}$ ( $U$ is the unit ball). Can $f$ be extended to a hyperfunction $u$ on $U$ such that $P(D) u=\sum_{\alpha} c_{\alpha} \partial^{\alpha} \delta_{0}$ where $\left\{c_{\alpha}\right\}_{\alpha \in I}$ satisfies condition $\mathrm{A}_{\varrho}$ for some $\varrho$ from $(* * *)$ ?

4.6. Remark. Corollary 10.8 .2 of [7] says that if $P(D)$ is elliptic then each open set in $\mathbb{R}^{n}$ is $P$-convex for supports. Hence Theorem 4.3 gives us an alternative approach to Theorems 3.1-3.4.

4.7. REMARK. Little is known about the isolated singularities of solutions of $P(D) u=0$ in the case when $P(D)$ is not hypoelliptic. Palamodov [8] proved that if $P(D) u=0$ on $U \backslash\{0\}$ and $u \in C^{\infty}(U \backslash\{0\}) \cap D^{\prime}(U)$ then $u \in C^{\infty}(U)$. He proved, however, that there can exist isolated essential singularities (that means solutions which cannot be extended to a distribution on $U)$. There are also some papers on isolated singularities of solutions of the Schrödinger equation. However, by Palamodov's result, the fundamental solution $E$ cannot have an isolated singularity at zero and thus we cannot have Laurent expansions of the type described above.

\section{References}

[1] N. Aronszajn, T. Creese and L. Lipkin, Polyharmonic Functions, Clarendon Press, Oxford, 1983. 
[2] S. Axler, P. Bourdon and W. Ramey, Harmonic Function Theory, Springer, 1992.

[3] M. Brelot, Eléments de la théorie classique du potentiel, 2-ème éd., Paris, 1961.

[4] S. Y. Chung, D. Kim and J. R. Lee, Generalized Bôcher's theorem, J. Math. Anal. Appl. 188 (1994), 341-345.

[5] S. J. Gardiner, Harmonic Approximation, London Math. Soc. Lecture Note Ser. 221, Cambridge Univ. Press, 1995.

[6] R. Harvey and J. C. Polking, A Laurent expansion for solutions to elliptic equations, Trans. Amer. Math. Soc. 180 (1973), 407-413.

[7] L. Hörmander, The Analysis of Linear Partial Differential Operators I, II, Springer, 1983.

[8] V. P. Palamodov, Linear Differential Operators with Constant Coefficients, Nauka, Moscow, 1967 (in Russian).

[9] P. Schapira, Théorie des Hyperfonctions, Lecture Notes in Math. 126, Springer, 1970.

[10] N. N. Tarkhanov, Laurent expansions and local properties of solutions of elliptic systems, Sibirsk. Mat. Zh. 29 (6) (1988), 124-134 (in Russian).

[11] - , Laurent Series for Solutions of Elliptic Equations, Nauka, Novosibirsk, 1991 (in Russian).

[12] - , The Analysis of Solutions of Elliptic Equations, Kluwer, Dordrecht, 1997.

[13] M. Wachman, Generalized Laurent series for singular solutions of elliptic partial differential equations, Proc. Amer. Math. Soc. 15 (1964), 101-108.

Department of Mathematics, Informatics and Mechanics

Warsaw University

Banacha 2

02-097 Warszawa, Poland

E-mail: elig@hydra.mimuw.edu.pl 